quant-ph/9604002

Zürich University Preprint: ZU-TH-10/96

\title{
Multiphoton Ionization as Time-Dependent Tunneling
}

\author{
Klaus Ergenzinger* \\ Institut für Theoretische Physik \\ Universität Zürich \\ Winterthurerstr. 190, CH-8057 Zürich, Switzerland
}

April 3, 1996

\begin{abstract}
A new semiclassical approach to ionization by an oscillating field is presented. For a $\delta$-function atom, an asymptotic analysis is performed with respect to a quantity $h$, defined as the ratio of photon energy to ponderomotive energy. This $h$ appears formally equivalent to Planck's constant in a suitably transformed Schrödinger equation and allows semiclassical methods to be applicable. Systematically, a picture of tunneling wave packets in complex time is developped, which by interference account for the typical ponderomotive features of ionization curves. These analytical results are then compared to numerical simulations [1] and are shown to be in good agreement.
\end{abstract}

*e-mail: erg@physik.unizh.ch 


\section{Introduction}

In recent times, there has been a lot of effort dedicated to a better understanding of ionization by strong laser fields (reviews e.g. in [2, 3]), especially since the discovery of nonperturbative effects like above-threshold ionization ATI (for a review see «), the sensitivity of ionization rates, and stabilization in superintense fields [5, 6, 7]. But there succeeded no analytical solution, not even to the simplest model, i.e. an electron bound by an attractive $\delta$-potential in the presence of an oscillating electric field. One of the main reasons is that there exist two separate regions. Inside the atomic core, the binding potential is predominant, and outside, it is vice versa the electric field that dominates (this is also the main point that makes perturbation theory work so poorly). For both regions, the distinct propagators are known exactly, but they cannot be combined to solve the ionization problem exactly.

An appropriate way of addressing this problem nonperturbatively is the semiclassical method, which we will use to construct the semiclassical propagator for ionization of such a $\delta$-function atom. The choice of this model has three advantages: first, there is a clear distinction between inside and outside the atom, so that there exists no intermediate region. Second, there is only one bound state for the $\delta$-potential, so there arise no difficulties with intermediate resonances and induced resonances by AC-Stark shift as it happens in real atoms. In this point, our model resembles photodetachment of a negative ion [8], especially $\mathrm{H}^{-}$. Third, the exact problem can be reduced to a Volterra type integral equation in time, for which accurate numerical solutions can be computed [1] and allow precise tests.

The above described problem (as well as its more general settings in three dimensions with more realistic binding potentials) has been treated in the literature in several ways. The so-called Keldysh-Faisal-Reiss (KFR) approach [9, 10, 11] consists in expressing the exact propagator $\hat{U}$ in terms of the known Volkov propagator $\hat{U}^{V}$ [12] (for a free electron in the electromagnetic field) and of $V_{\delta}$, the atomic binding potential: $\hat{U}=\hat{U}^{V}+$ $\hat{U}^{V} V_{\delta} \hat{U}$. The unknown $\hat{U}$ on the right hand side is approximated by $\hat{U}^{V}$ (equivalent to Born approximation), and matrix-elements for ionization are calculated between the 
ground state and so-called Volkov-states [12] in the continuum. This approach has been extended and refined by many authors, and we will compare our results with two such typical extensions [13, 14].

Another approach, the so-called two-step model [15, 16, 17], clearly distinguishes between ionization first and classical propagation in the laser field afterwards. This proved to be very useful especially in calculating high-harmonic generation [18]. This separation into two steps will be used in the following, but now justified in a fully semiclassical context.

In addition, there exist several other approaches; a very common method is using Floquet theory [19, 20, 21], which explicitly incorporates the periodicity of the timedependent Hamiltonian.

Our issue here is not to obtain better results for a simple model, but to gain better physical insight into the mechanisms of ionization processes using semiclassical methods.

This paper is organized as follows: After basic definitions, we demonstrate characteristic elements of typical ionization curves which we want to understand semiclassically. Using a "sum over classical paths" technique, the total semiclassical propagator is constructed successively by identifying the paths which are relevant for ionization. First for the part remaining bound, and then for the free wave packets stemming from timedependent tunneling in complex time. From this semiclassical propagator, the total ionization rate is derived and compared to numerical simulations as well as to other theories in the literature. 


\section{Basics}

\subsection{Definition of the model}

We want to study the ionization of a one-dimensional $\delta$-function atom $V_{\delta}=-\alpha \delta(x)$, which possesses exactly one bound state with energy $E_{0}=-\alpha^{2} / 2$ (for the threedimensional analog cf. [22]). This atom is exposed to an oscillating electric field $V_{0}=-\mu x \cos (\omega t)$ in the so-called dipole-approximation. The parameter $\alpha$ stands for the strength of the binding potential, $\mu$ for the amplitude of the applied electric field, and $\omega$ for its angular frequency. The Schrödinger equation in atomic units $(\hbar=m=1)$ is

$$
i \frac{\partial}{\partial t} \psi=\left(-\frac{1}{2} \frac{\partial^{2}}{\partial x^{2}}-\alpha \delta(x)-\mu x \cos (\omega t)\right) \psi
$$

From these three parameters, there can be derived (due to the scaling properties of (1)) two independent, meaningful, and dimensionless quantities:

first, there is $z$, the ratio of ponderomotive energy $U_{\text {pond }}=\mu^{2} /\left(4 \omega^{2}\right)$ to photon energy $\omega: z=\mu^{2} /\left(4 \omega^{3}\right)$. $U_{\text {pond }}$ is the mean kinetic energy of a free electron in an oscillating field.

and second the so-called Keldysh-factor [9] $\gamma=\alpha \omega / \mu$, which is the ratio of the (adiabatic) tunneling time to the period of the applied field. This $\gamma$ characterizes the ionization process; $\gamma \ll 1$ corresponds to (adiabatic) tunneling, and $\gamma \gg 1$ is better described in a pure multiphoton frame [23, 24].

These are the two quantities by which the model will be described below. A third, but no more independent quantity is $n_{i o}$, the ratio of necessary ionization energy $E_{0}$ to photon energy:

$$
n_{i o}=\alpha^{2} /(2 \omega)=2 \gamma^{2} z
$$




\subsection{Transformation of the Schrödinger equation}

Using the following coordinate and time transformation, we cast the above Schrödinger equation (11) in a very suitable form:

$$
x^{\prime}=\frac{\omega^{2}}{\mu} x, t^{\prime}=\omega t
$$

Using the scaling relation $\delta(a x)=\delta(x) /|a|$, which is identical to the scaling behaviour of the Coulomb-potential, and omitting the primes, we arrive at:

$$
i h \frac{\partial}{\partial t} \psi=\left(-\frac{1}{2} h^{2} \frac{\partial^{2}}{\partial x^{2}}-h \gamma \delta(x)-x \cos (t)\right) \psi
$$

Here $h:=\omega^{3} / \mu^{2}=1 /(4 z)$ is written very suggestively to indicate that we have obtained a parameter $h$ formally identical to Planck's constant $\hbar$ in ordinary quantum mechanics using SI units. Of course we can give $h$ any value we like. Restricting ourselves to strong fields where $U_{\text {pond }} \gg \omega$, i.e. $z \gg 1, h$ can get arbitrarily small. This will allow us to use the normal semiclassical methods, exploiting the formal analogy between the parameter $h$, introduced above, and Planck's constant $\hbar$. Still remarkable is the appearance of the factor $h$ in front of the binding $\delta$-potential, its implications in the semiclassical limit will be derived below.

The (normalized) ground state wavefunction for the $\delta$-function atom without applied external field is

$$
\begin{aligned}
\psi_{0}(x) & =\sqrt{\frac{\gamma}{h}} \exp \left(-\frac{\gamma}{h}|x|\right) \\
\hat{H}_{0} \psi_{0} & =-\frac{\gamma^{2}}{2} \psi_{0} \\
\psi_{0}(x, t) & =\psi_{0}(x) \exp \left(i \frac{\gamma^{2}}{2 h} t\right),
\end{aligned}
$$

which leads to the stronger a localization the smaller $h$ is. In the limiting case $h \rightarrow 0$, $\psi_{0}(x)$ approaches (appropriately scaled) the spatial Dirac $\delta$-function:

$$
\frac{1}{2} \sqrt{\frac{\gamma}{h}} \psi_{0}(x) \rightarrow \delta(x)
$$

In the following, this approximation will be used only for calculation of scalar products, so that no mathematical ambiguities will arise. 


\section{Prominent Semiclassical Features in Ionization Rates}

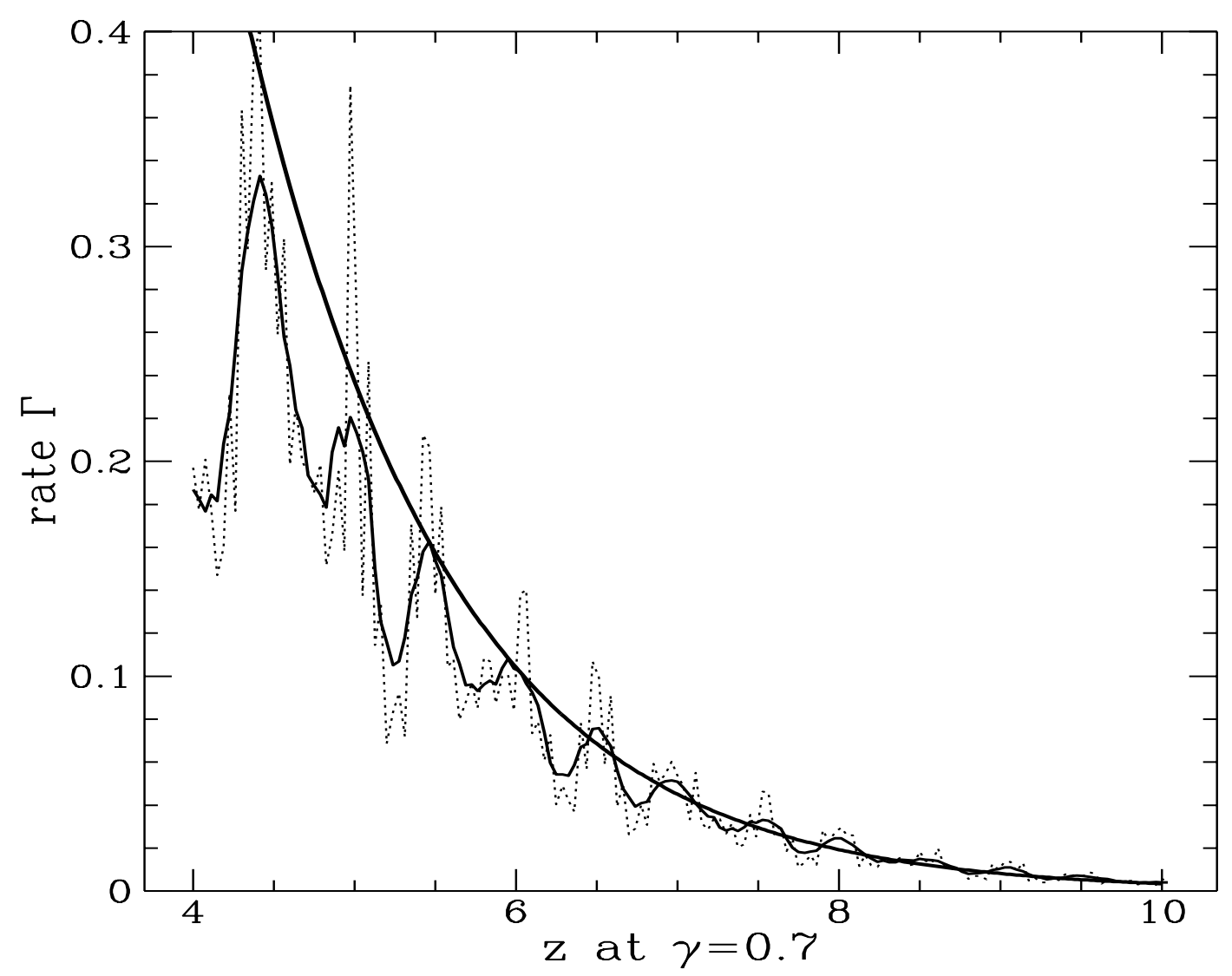

Figure 1: Numerical ionization rates, raw (dotted) and smoothed (thicker) versus number of ponderomotive photons $z$, compared with WKB-background (thick, smooth decaying curve). The Keldysh parameter $\gamma$ and not the depth of the binding potential is kept fixed.

The ionization rates from a numerical analysis [1] of the model show certain characteristic features. In figure 1, we see the raw results (dotted), these results smoothed (thicker) and the adiabatically averaged WKB-value (see eq. (16), depicted thick, monotonically decreasing). The ionization rate $\Gamma$ is shown against $z$, the ponderomotive energy (over photon energy). The WKB-rate accounts well for the background, but on the actual rate, there is a periodic modulation and a lot of fine-structure super- 
posed. The (slow) modulation becomes obvious after smoothing the raw data (using a Savitzky-Golay filter technique [25]) in order to eliminate the fine structure and to work out this fundamental modulation.

The period of this modulation can be understood by so-called channel closing arguments [26], but there is no argument for the amplitude of modulation. The $k-$ th channel means a multiphoton ionization by $k$ photons with energy balance (here in atomic units)

$$
k \omega=n_{i o} \omega+z \omega+E_{k i n}
$$

$n_{i o} \omega$ is the energy required for ionization, $z \omega$ is the (ponderomotive) energy $U_{\text {pond }}$ contained in the electron oscillation in the electric field, and $E_{k i n}$ is the additional kinetic energy the free electron gains in this ionization process. This channel is energetically only allowed for $E_{k i n} \geq 0$, otherwise it is forbidden.

The threshold for the $k$-th channel is defined using the condition $E_{k i n}=0$. In terms of $\gamma$ and $z$, equation (8) at threshold is

$$
k=2 \gamma^{2} z_{k}+z_{k}
$$

and the specific value $z_{k}$ at threshold becomes

$$
z_{k}=\frac{k}{1+2 \gamma^{2}}
$$

yielding a period $\Delta z=1 /\left(1+2 \gamma^{2}\right)$ in $z$, i.e. the period of approximately one half in the example of figure 11.

An important point to note is that the numerical results give strong evidence for regular behaviour at threshold, whereas the usual prediction of appropriate theories (e.g. those of KFR type) is a divergence at threshold.

Here it is important to note that the features described above are definitely not restricted only to the $\delta$-function atom. Numerical simulations for various model potentials in the literature exhibit similar features, often with remarkable quantitative correspondence (see figure 8 in [1], comparing the $\delta$-function atom to a smoothed 
binding potential $V(x)=-\exp (-|x|) / \sqrt{x^{2}+x_{0}^{2}}$ as used by Greenwood and Eberly [27]).

In the following, a semiclassical theory is derived that accounts for the information contained in smoothed rates. The WKB-background as well as the periodicity, amplitude, and phase of modulation are contained in a single, divergence free theory, which constructs the propagator using the semiclassical sum over paths, cf. e.g. 28.

\section{Quasi-Energies}

\subsection{Calculation of WKB-coefficient}

The main effect of applying an external field to an atom is that the bound state becomes metastable and tunneling can occur. In the static case, this tunneling rate $D$ can be approximated using the normal WKB-coefficient for the corresponding barrier. For a linear potential barrier $V(x)=-\eta x$ and a given (negative) energy $E_{0}$, the well-known expression for $D$ is

$$
D(\eta) \approx \exp \left(-\frac{2}{h} \int_{0}^{-E_{0} / \eta}|p(x)| d x\right)
$$

Using the (imaginary) local momentum $p(x)=\sqrt{2\left(E_{0}+\eta x\right)}$ and the ground state energy $E_{0}=-\gamma^{2} / 2$, this evaluates to

$$
D(\eta) \approx \exp \left(-\frac{2}{3} \frac{\gamma^{3}}{\eta h}\right)
$$

Of course, this approach gives only the exponential part, but since the preexponential part is well-known from the literature for this simple case (e.g. [14, 29]), we can adopt it from there. This factor is just twice the atomic frequency, in our units $2 E_{0} / h$

$$
D(\eta)=\frac{\gamma^{2}}{h} \exp \left(-\frac{2}{3} \frac{\gamma^{3}}{\eta h}\right)
$$

If we consider a time-dependent external field, the parameter $\eta$ becomes time-dependent too and represents the instantaneous strength of the applied field at the moment the 
ionization takes place: $\eta=|\cos (t)|$. If the tunneling process occurs on a much shorter time scale than the period $2 \pi$ of the oscillation, it is a good idea to consider the ionization taking place adiabatically, i.e. calculate the instantaneous ionization rate $D(|\cos (t)|)$ and average it over a whole period. This case corresponds to $\gamma \ll 1$, i.e. the Keldysh factor characterizing the ionization is quite small.

In calculating the cycle-average $\bar{D}$ over a cosine period, one has to integrate and to normalize subsequently

$$
\bar{D}=\frac{1}{2 \pi} \int_{0}^{2 \pi} \frac{\gamma^{2}}{h} \exp \left(-\frac{2}{3} \frac{\gamma^{3}}{|\cos (t)| h}\right) d t
$$

Because we want to examine the asymptotic case $h \rightarrow 0$, we best evaluate this integral using the method of steepest descent (also called saddle-point integration, cf. appendix A) with respect to $h$. The derivation of the exponential argument with respect to time $t$ gives

$$
\frac{d}{d t}\left(-\frac{2}{3} \frac{\gamma^{3}}{\cos (t)}\right)=-\frac{2}{3} \gamma^{3} \frac{\sin (t)}{\cos (t)^{2}}
$$

The relevant times are the zeros of this expression, i.e. all multiples of $\pi$. These are the instants where the electric field strength $|\cos (t)|$ is a maximum. For symmetry reasons, all these instants are equivalent and it is sufficient to evaluate the above integral around one such instant using the method of steepest descent. The result is

$$
\begin{aligned}
\bar{D} & =\sqrt{\frac{3 h}{\pi \gamma^{3}}} \frac{\gamma^{2}}{h} \exp \left(-\frac{2}{3} \frac{\gamma^{3}}{h}\right) \\
& =\sqrt{\frac{3 h}{\pi \gamma^{3}}} D(\eta=1)
\end{aligned}
$$

This shows that the average $\bar{D}$ is the instantaneous ionization rate at maximum field strength, up to a preexponential factor.

Note that the method of steepest descent becomes exact in the limit of vanishing $h$. This allows a very interesting interpretation. In this case, the ionization effectively takes place only in the vicinity of the instants of maximal field strength $\eta=1$. This means that there exist ionization bursts, separated by half a period, between which practically no other ionization occurs. In the following, this property will be used to 
construct a scenario of propagating wave packets, emerging from these peak instants, freely propagating afterwards, and interfering with one another and with the part of the wave function remaining bound.

\subsection{AC-Stark effect}

The second effect of applying an external field is the so-called Stark effect, in the oscillating time-dependent case called AC-Stark effect . We will treat this effect adiabatically as well (cf. [30]), the well-known value (e.g. [14, 29, 31]) for the instantaneous energy-shift $E^{A C}$ is $E^{A C}(\eta)=-5 h^{2} \eta^{2} /\left(8 \gamma^{4}\right)$. Cycle-averaging results in

$$
\bar{E}^{A C}=\frac{1}{2} E^{A C}(\eta=1)=-\frac{5 h^{2}}{16 \gamma^{4}}
$$

This effect means an additional phase factor in the propagator $\exp (-i \hat{H} t / h)$, whereas the tunneling rate, calculated by taking absolute squares of the wave function, is not affected by $E^{A C}$. Note that the influence of (18) will be quite small in the following because of its proportionality to $h^{2}$.

In order to express tunneling and Stark shift together, it is useful to write the exponential decay of the bound state using an imaginary contribution $E^{I}$ to the total energy $E^{m}$. Setting

$$
\begin{aligned}
E^{I}(\eta) & =-i \frac{\gamma^{2}}{2} \exp \left(-\frac{2}{3} \frac{\gamma^{3}}{h \eta}\right)=-\frac{i h}{2} D(\eta) \\
E^{m}(\eta) & =E_{0}+E^{A C}(\eta)+E^{I}(\eta)
\end{aligned}
$$

the adiabatic development of the ground state can be described by the propagator

$$
\hat{U}^{\delta}\left(t_{f}\right)=\exp \left(-\frac{i}{h} \int_{0}^{t_{f}} E^{m}(|\cos (t)|) d t\right) \hat{P}_{0}
$$

using the total quasi-energy $E^{m}$ and the projection operator $\hat{P}_{0}$, projecting onto the ground state. This adiabatic description is useful if we want to describe the propagation of the wavefunction for arbitrary times. If we restrict ourselves to considering only full cycles, we can use the appropriate averages.

$$
\bar{E}^{I}=\sqrt{\frac{3 h}{\pi \gamma^{3}}} E^{I}(\eta=1)
$$




$$
\begin{aligned}
\bar{E}^{m} & =E_{0}+\bar{E}^{A C}+\bar{E}^{I} \\
\hat{\bar{U}}^{\delta}\left(t_{f}\right) & =\exp \left(-\frac{i}{h} \bar{E}^{m} t_{f}\right) \hat{P}_{0}
\end{aligned}
$$

Applying this propagator to the ground state and taking absolute squares results just in the exponential decay with rate $\bar{D}$. This will be sufficient for the forthcoming considerations. For $t_{f}=2 k \pi, k=0,1,2, \ldots$, both propagators are of course identical, due to the very construction of the average.

When comparing these expressions with the numerical results described later, we will see that they can account for the monotonic background of the ionization rate (see figure 1), but if we want to explain the quasi-periodic modulations, responsible for the nonmonotonicity of the rate, we have to go further in our semiclassical description.

\section{Semiclassical Propagators}

\subsection{General construction}

To construct the semiclassical propagator outside the binding potential, we start with the (formal) path integral expression

$$
\begin{aligned}
U\left(x, t_{f} ; y, t_{i}\right) & =\int_{t_{i}}^{t_{f}} \mathcal{D} x(t) \exp \left(\frac{i}{h} S[x(t)]\right) \\
S[x(t)] & =\int_{t_{i}}^{t_{f}} L(x(t), \dot{x}(t)) d t \\
L & =T-V=L_{0}-V_{\delta}=T-V_{0}-V_{\delta}
\end{aligned}
$$

Here $T$ is the kinetic energy operator, $L$ is the full Lagrangian, and $L_{0}$ is the Lagrangian for the electric field $V_{0}$ alone, with the binding potential $V_{\delta}$ excluded. The usual procedure in the semiclassical limit $h \rightarrow 0$ is to find the stationary paths with $\delta S=0$, yielding the classical paths by means of the Euler-Lagrange equation [32]. The remarkable point here is that $V_{\delta}=-h \gamma \delta$ contains a factor $h$. This becomes important for the semiclassical limit, because this $h$ cancels in the exponent $i S / h$, and consequently this 
part of the phase does no more fluctuate arbitrarily fast for non-stationary paths in the semiclassical limit.

Applying the analogy to saddle-point integration in function space (cf. appendix A), we notice that we only have to vary $S_{0}=\int L_{0}$ in order to find the stationary paths to $\delta S_{0}=0$. This condition gives the classical path $x_{c l}(t)$ to $L_{0}$ by means of the EulerLagrange equation

$$
\frac{d}{d t}\left(\frac{\partial}{\partial \dot{x}} L_{0}\right)-\frac{\partial}{\partial x} L_{0}=0
$$

subject to the boundary conditions imposed by the path integral.

$$
\begin{aligned}
x_{c l}\left(t_{i}\right) & =y, x_{c l}\left(t_{f}\right)=x \\
x_{c l}(t) & =x_{c l}\left(t \mid x, t_{f} ; y, t_{i}\right) \\
& =-\cos (t)+\cos \left(t_{i}\right)+y+\frac{x-y+\cos \left(t_{f}\right)-\cos \left(t_{i}\right)}{t_{f}-t_{i}}\left(t-t_{i}\right)
\end{aligned}
$$

$V_{\delta}$ in the full Lagrangian $L$ only accounts for an additional phase factor $\exp (i \gamma \phi)$ to the propagator

$$
\begin{aligned}
\phi & =\int_{t_{i}}^{t_{f}} \delta\left(x_{c l}(t)\right) d t \\
& =\sum_{t_{0}^{j}} 1 /\left|\dot{x}_{c l}\left(t_{0}^{j}\right)\right|
\end{aligned}
$$

where the $t_{0}^{j}$ denote the zeros of the classical path $x_{c l}\left(t_{0}^{j}\right)=0$. This phase $\phi$ jumps every time the classical path $x_{c l}\left(t \mid x, t_{f} ; y, t_{i}\right)$ crosses the $\delta$-potential at the origin.

The result for the semiclassical propagator $U^{s c}$ is

$$
\begin{aligned}
U^{s c}\left(x, t_{f} ; y, t_{i}\right) & =\frac{1}{\sqrt{2 \pi i h}} \sqrt{-\frac{\partial^{2}}{\partial x \partial y} S_{0}} \exp \left(\frac{i}{h} S_{0}\right) \exp (i \gamma \phi) \\
& =\frac{1}{\sqrt{2 \pi i h\left(t_{f}-t_{i}\right)}} \exp \left(\frac{i}{h} \int_{t_{i}}^{t_{f}} L_{0}\left(x_{c l}(t), \dot{x}_{c l}(t)\right) d t\right) \exp (i \gamma \phi)
\end{aligned}
$$

The same result is derived in appendix $\mathrm{B}$ using the WKB-ansatz.

In general, one would have to include so-called Maslov phase factors [33, but we can omit them because we do not encounter any caustics in this problem. Since $V_{0}=$ 
$-x \cos (t)$ is linear in $x$, the appropriate semiclassical propagator for $L_{0}$ is identical [34] to the exact one, namely the well-known Volkov propagator $U^{V}$ [12]. The result can now be understood as the Volkov propagator $U^{V}$ plus additional phase jumps for every crossing of the origin.

$$
U^{s c}=U^{V} \exp (i \gamma \phi)
$$

\subsection{Special tunneling propagator}

There is one important point to note; the description of the propagator using regular classical paths is only justified after the electron has tunneled out. So in order to describe the tunneling paths, which classically do not exist, one has to modify the above description; a common method is to introduce complex time and coordinates (cf. appendix 9 and [35, 36]).

In our case, we know the bound state $\psi_{0}=\sqrt{\gamma / h} \exp (-\gamma|x| / h)$, which formally equals a free wave $\exp (i p x / h)$ with complex momentum $p_{0}= \pm i \gamma$. This is consistent with a negative energy $E=p_{0}^{2} / 2=-\gamma^{2} / 2$, which is just the ground state energy $E_{0}$ of the $\delta$-potential.

We choose the following complex boundary conditions (a similar reasoning appeared in [37) for the complex tunneling path $x_{T}$ :

the initial momentum (imaginary part Im considered only)

$$
\operatorname{Im}\left(\dot{x}_{T}\left(t_{0}\right)\right)=p_{0}=+i \gamma
$$

(plus sign chosen to ensure exponential decay of wavefunctions and not growth), and the initial position

$$
x_{T}\left(t_{0}\right)=y
$$

Additionally, $x_{T}$ must fulfill the final condition

$$
x_{T}\left(t_{f}\right)=x
$$


which is the boundary condition at the end of the path. The additional free constant $t_{0}$ is necessary because we impose three boundary conditions. But the ordinary differential equation (28), which $x_{T}$ must obey, is of order two, and therefore has only two free constants.

The idea is that tunneling takes place in the imaginary part between $t=t_{0}$ and $t=t_{f}$, and free propagation $U^{s c}$ (under the influence of the oscillating electric field) takes place vice versa in the real part. This interpretation is allowed by the usual decomposition rules for semiclassical operators (cf. [38, 39]).

The general expression for such a path $x_{T}$ fulfilling the (now complex-valued) equation of motion (28) is

$$
\begin{aligned}
& x_{T}(t)=-\cos (t)+\cos \left(t_{0}\right)+y+v_{0}\left(t-t_{0}\right) \\
& \dot{x}_{T}(t)=\sin (t)+v_{0}
\end{aligned}
$$

The first condition $\operatorname{Im}\left(\dot{x}_{T}\left(t_{0}\right)\right)=p_{0}$ yields

$$
i \gamma=\operatorname{Im}\left(\sin \left(t_{0}\right)+v_{0}\right)
$$

Now we see the meaning of $t_{0}$; it must account for the complex boundary condition and so we set

$$
t_{0}=i \operatorname{arcsinh}(\gamma)
$$

in order to allow $v_{0}$ to remain real (see also [37 for this result). The second boundary condition (38) is fulfilled trivially by the ansatz (40), and from the third conditon we obtain

$$
v_{0}=\frac{x-y+\cos \left(t_{f}\right)-\cos \left(t_{0}\right)}{t_{f}-t_{0}}
$$

Using the equations (40,43,44), we can easily construct the complete propagator $U^{T}$ containing tunneling as well as propagation in the electric field. The result is just the analytic continuation of our former result $U^{s c}$ in eq. (35)

$$
U^{T}\left(x, t_{f} ; y, t_{0}\right)=\frac{1}{\sqrt{2 \pi i h\left(t_{f}-t_{0}\right)}} \exp \left(\frac{i}{h} \int_{t_{0}}^{t_{f}} L_{0}\left(x_{T}(t), \dot{x}_{T}(t)\right) d t\right) \exp \left(i \gamma \phi\left[x_{T}\right]\right)
$$


Note that the argument of the square root in the denominator is now truely complex, so that we have an ambiguity in choosing a certain sheat of the complex root. We decide to define $\sqrt{r \exp (i \varphi)}=-\sqrt{r} \exp (i \varphi / 2), \varphi \in[0,2 \pi]$. Note further that the tunneling starts at $t_{0}=i \operatorname{arcsinh}(\gamma)$, and in order to reach $t_{0}$, we first have to propagate the ground state from $t=0$ to $t_{0}$ using $\hat{\bar{U}}^{\delta}\left(t_{0}\right)$, the analytic continuation in time of (24). This path in the complex $t$-plane is depicted in figure 2.

According to the composition rule for propagators, the complete propagator $\hat{U}^{c}$ for the ground state from $t=0$ to $t_{f}$ is

$$
\hat{U}^{c}\left(t_{f}, 0\right)=\hat{U}^{T}\left(t_{f}, t_{0}\right) \hat{\bar{U}}^{\delta}\left(t_{0}\right)
$$

or in coordinate representation

$$
\hat{U}^{c}\left(x, t_{f} ; y, 0\right)=\int_{-\infty}^{+\infty} \hat{U}^{T}\left(x, t_{f} ; z, t_{0}\right) \hat{\bar{U}}^{\delta}\left(z, t_{0} ; y, 0\right) d z
$$

\subsection{Generalization to other ionization bursts}

The result of the previous section can be easily generalized to later ionization bursts. Figure 2 shows in the complex $t$-plane that the electron does not become instantaneously free, but rather propagates from $t=0$ to $t=t_{0}$ according to the propagator $\hat{\bar{U}}^{\delta}\left(t_{0}\right)$ (valid inside the binding potential), and then tunnels and propagates from $t_{0}$ to $t_{f}=2 \pi$ according to the complex-valued propagator $\hat{U}^{T}$ valid outside. The above line integrals over the depicted paths are path-independent [35], so there does not exist a unique path. The important point is the propagation with different propagators from distinct starting points to distinct endpoints. This describes the first ionization burst, but in order to describe the wave packets emerging from the bursts at times $t_{k}=k \pi, k=0,1,2,3, \ldots, k \pi<t_{f}$, one can repeat the above calculations.

The propagator $\hat{U}_{k}^{c}\left(t_{f}, 0\right)$ for the wave packet stemming from $t=k \pi$ is just

$$
\hat{U}_{k}^{c}\left(t_{f}, 0\right)=\hat{U}^{T}\left(t_{f}, k \pi+t_{0}\right) \hat{\bar{U}}^{\delta}\left(k \pi+t_{0}\right)
$$

In this notation, $\hat{U}_{0}^{c}$ is identical to the above $\hat{U}^{c}$. The interpretation is that the electron remains trapped from $t=0$ to $t=k \pi$ and then propagates into the complex $t$-plane up 


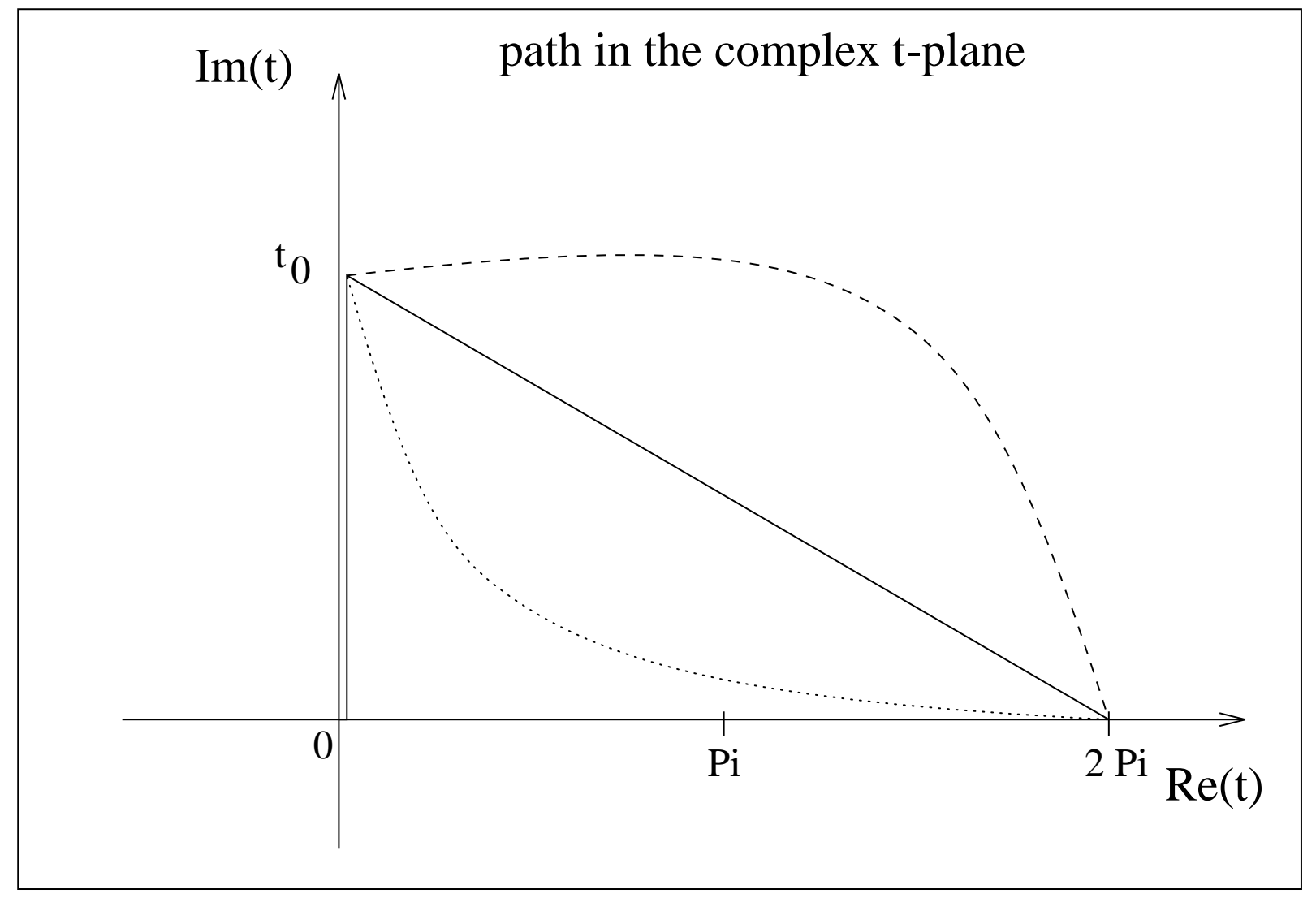

Figure 2: Path in the complex $t$-plane, describing the evolution of the first wave packet stemming from $t=0$. Since the line integral for the calculation of the semiclassical propagator is path-independent, these paths can be chosen at random. Important is that the propagation from $t=0$ to $t=t_{0}$ uses another propagator $\hat{\bar{U}}^{\delta}$ than the propagator $\hat{U}^{T}$ afterwards to $t_{f}=2 \pi$.

to $t=k \pi+t_{0}$, both according to $\hat{\bar{U}}^{\delta}$. It then tunnels and propagates from $t=k \pi+t_{0}$ up to $t=t_{f}$, according to $\hat{U}^{T}$ (45), i.e. the (complexified) Volkov propagator plus phase jumps. Figure 3 contains the four paths corresponding to four wave packets created at $t=0, \pi, 2 \pi, 3 \pi$, which interfere at $t_{f}=4 \pi$. Once again, these line integrals are path-independent so that one can choose these paths at random. 


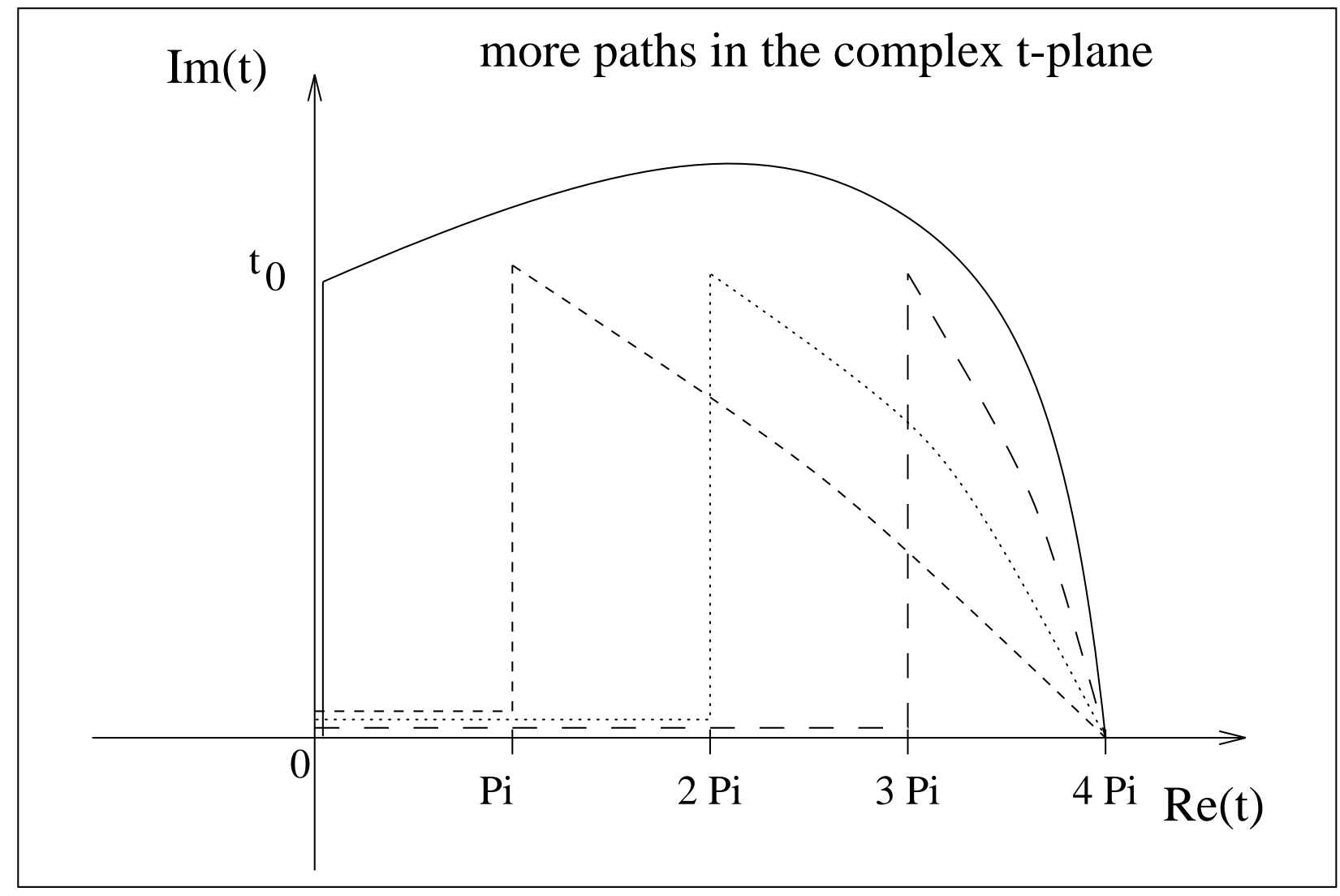

Figure 3: The various paths in the complex $t$-plane for four wave-packets are depicted. The path for the wave packet emerging at $k \pi$ goes from 0 to $k \pi+t_{0}$ and afterwards to $t_{f}=4 \pi$. Again we have path-independence for the two parts.

\section{Interference between Paths}

The electron has two possibilities, it can tunnel and propagate as well as remain bound by the binding potential. The quantum mechanical amplitudes for both processes are known and they can be added in order to obtain a better description of the evolution of the system. This is just the "semiclassical sum over classical paths" method, e.g. [28.

The full propagator $\hat{U}$ is the sum of the propagator $\hat{\bar{U}}^{\delta}$, valid for the part bound by the $\delta$-potential, and the $\hat{U}_{k}^{c}$ 's, the propagators for tunneling at $t=k \pi$ and (free) 
propagation afterwards.

$$
\hat{U}\left(t_{f}, 0\right)=\hat{\bar{U}}^{\delta}\left(t_{f}\right)+\sum_{k=0,1,2, \ldots} \hat{U}_{k}^{c}\left(t_{f}, 0\right)
$$

\subsection{First period}

For simplicity and notational reasons, we will first consider only the wave packet originating from the ionization burst at $t=0$. The other propagators can be added as well and the resulting integrals can be evaluated using the same techniques as described below. We will restrict ourselves to examining the wave function after full periods $t_{f}=2 n \pi$, and we will deal mostly with just one period. The second burst occuring at $t=\pi$ during the first period is of secondary importance, because this free electron follows a classical trajectory $x_{c l}=a+b(t-\pi)-1-\cos (t)$ and its center is about -2 to the left at $t_{f}=2 n \pi$. Therefore the overlap of this wave packet with the ground state $\psi_{0}$ can be neglected.

This effect as well as the influence of considering several periods and wave packets will be demonstrated, when evaluating the analytic expressions derived below and comparing them to numerical results in section 7.1 .

This propagator (49) must be applied to the ground state $\psi_{0}$ (5) in order to obtain the wavefunction $\psi\left(t_{f}\right)$ at a certain time $t_{f}$

$$
\psi\left(t_{f}\right)=\hat{U}\left(t_{f}, 0\right) \psi_{0}
$$

Applying the propagator $\hat{\bar{U}}^{\delta}(t)$ results in just a phase factor $\exp \left(-\left(i \bar{E}^{m} / h\right) t\right)$, and so the above expression (50) simplifies to (for one period $t_{f}=2 \pi$ )

$\psi\left(x, t_{f}\right)=\exp \left(-\frac{i \bar{E}^{m}}{h} t_{f}\right) \psi_{0}(x)+\exp (i \gamma \phi) \exp \left(-\frac{i \bar{E}^{m}}{h} t_{0}\right) \int_{-\infty}^{+\infty} U^{V}\left(x, t_{f} ; y, t_{0}\right) \psi_{0}(y) d y(51)$

Using the property (7) that $\psi_{0}(y)$ approaches the spatial $\delta$-function in the semiclassical limit $h \rightarrow 0$, the integral can be evaluated to

$$
\psi\left(x, t_{f}\right)=\exp \left(-\frac{i \bar{E}^{m}}{h} t_{f}\right) \psi_{0}(x)+\exp (i \gamma \phi) \exp \left(-\frac{i \bar{E}^{m}}{h} t_{0}\right) 2 \sqrt{\frac{h}{\gamma}} U^{V}\left(x, t_{f} ; 0, t_{0}\right)(52)
$$


In order to calculate the probability amplitude $p$ for the electron to remain bound, one has to project onto the ground state $\psi_{0}(x)$

$$
p=\int_{-\infty}^{+\infty} \psi\left(x, t_{f}\right) \psi_{0}^{*}(x) d x
$$

Using the spatial localization property (7) again, this simplifies to

$$
p=\exp \left(-\frac{i \bar{E}^{m}}{h} t_{f}\right)+\exp \left(-\frac{i \bar{E}^{m}}{h} t_{0}\right) 4 \frac{h}{\gamma} U^{V}\left(0, t_{f} ; 0, t_{0}\right)
$$

$\bar{E}^{m}$ and $t_{0}$ are known from the equations (23) and (43), respectively. The phase $\phi$ is identical to 0 because the relevant classical path $x_{c l}=1-\cos (t)$ never crosses the origin. The first part of this expression for $p$ clearly accounts for the background, while the second part determines the amplitude, phase, and period of the superposed (slow) modulation.

\subsection{Fundamental periodicity}

The phase of the first term in eq. (54) is mainly given by the expression $-E_{0} t_{f} / h$, and the phase of the second term is dominated by the action $S^{c l} / h$ along the classical path $x_{c l}=1-\cos (t)$.

$$
S^{c l}=\int_{0}^{t_{f}}\left(\dot{x}_{c l}^{2}(t)+x_{c l}(t) \cos (t)\right) d t=-z t_{f}
$$

The last identity is straight forward (also in atomic units). Combining the phases and comparing to multiples of $2 \pi$ results (for $t_{f}=2 \pi$ ) exactly in equation (8) with $E_{k i n}=0$. This is just the threshold condition for channel closing, and implies the same periodicity. The period in $z$ is $\Delta z=1 /\left(1+2 \gamma^{2}\right)$, if $\gamma$ is kept fixed, or a period of $\Delta z=1$, if the depth of the binding potential $\alpha$ is kept fixed (see figure 0 later).

\subsection{More periods}

Again this result can be generalized easily to more bursts and longer final times $t_{f}>2 \pi$. Be $t_{f}=2 n \pi$, then one has to sum over $2 n$ bursts and amplitudes, and (this time 
written explicitly) the result for $p$ is

$$
\begin{aligned}
p= & \exp \left(-\frac{i}{h} \bar{E}^{m} t_{f}\right)+\sum_{k=0}^{2 n-1} \frac{-4 h}{g \sqrt{2 i \pi h\left(t_{f}-t_{0}-k \pi\right)}} \exp \left(\zeta^{k}\right) \\
\zeta^{k}= & \frac{-i}{4 h\left(t_{f}-t_{0}-k \pi\right)}\left(\left(t_{0}+k \pi\right)^{2}+\left(t_{f}-t_{0}-k \pi\right) \cos \left(t_{0}\right) \sin \left(t_{0}\right)\right. \\
& \left.-2\left(t_{0}+k \pi\right) t_{f}+4 \cos \left(t_{0}\right)(-1)^{k}-2+t_{f}^{2}-2 \cos ^{2}\left(t_{0}\right)\right)-\frac{i}{h} \bar{E}^{m}\left(t_{0}+k \pi\right)
\end{aligned}
$$

Note the useful relations $\cos \left(t_{0}\right)=\sqrt{1+\gamma^{2}}$ and $\sin \left(t_{0}\right)=i \gamma$. Propagating the system for longer times $t_{f}>2 \pi$ means to have more phase built up in the exponents of (56), and results in a finer resolution in the ionisation rate $\Gamma$; higher frequencies than the basic modulation period can be accounted for. How this can explain the fine-structure is demonstrated later on in figure 6 . 
In the above sum (56), only the contributions with $k$ even are important. This is because the centers of wave packets stemming from $k$ odd are located at about -2 to the left at $t_{f}=2 n \pi$, and therefore the overlap is very small.

\subsection{Ionization rate}

The probability $w$ for not being ionized is now calculated by taking the absolute square $w=|p|^{2}$, and the corresponding ionization rate $\Gamma$, fitting the exponential decay $w=$ $\exp \left(-\Gamma t_{f} /(2 \pi)\right)$, can be defined as

$$
\Gamma=-\frac{2 \pi}{t_{f}} \ln \left(|p|^{2}\right)
$$

For this quantity $\Gamma$, we will now compare the above semiclassical results with those from numerical simulations [1]. 


\section{Comparisons}

\subsection{Comparison with numerical results}

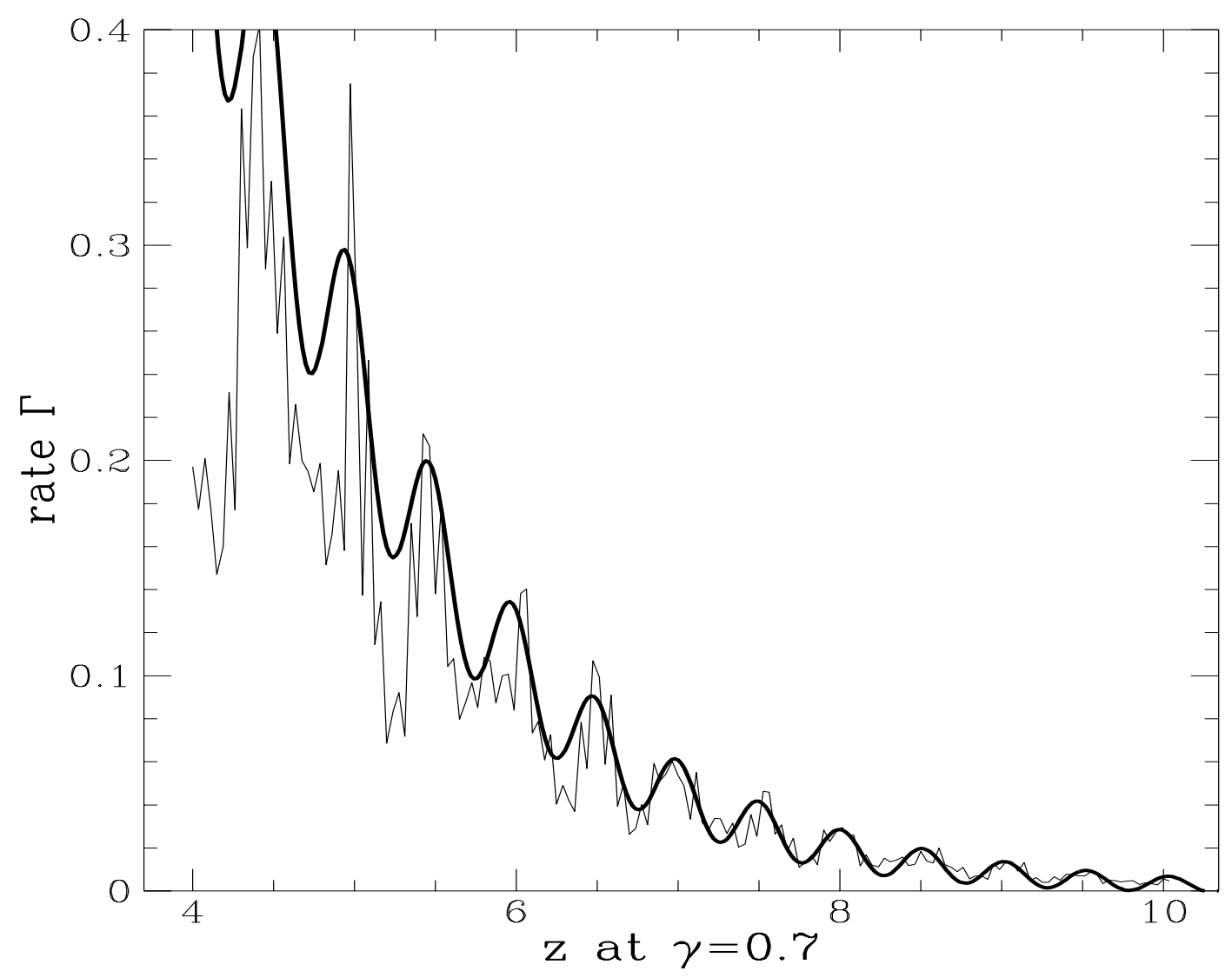

Figure 4: Numerical ionization rate (thin and jagged) and semiclassical approximation (thick and smooth) versus number of ponderomotive photons $z$ for $\gamma=0.7$

The numerical results are obtained by an integral equation method implemented by K. Sonnenmoser (《1] for details), which allows high-resolution scans and exhibits a lot of fine structure. While $\gamma$ is kept fixed, $z$ is varied, and so the semiclassical limit $h \rightarrow 0$ corresponds just to $z \rightarrow \infty$. This means that the agreement will become the better the larger $z$ is.

The interesting region for $\gamma$ is of course $\gamma \approx 1$, because this is the transition region between adiabaticity and multiphoton regime. For $\gamma \ll 1$, the adiabaticity criterion 


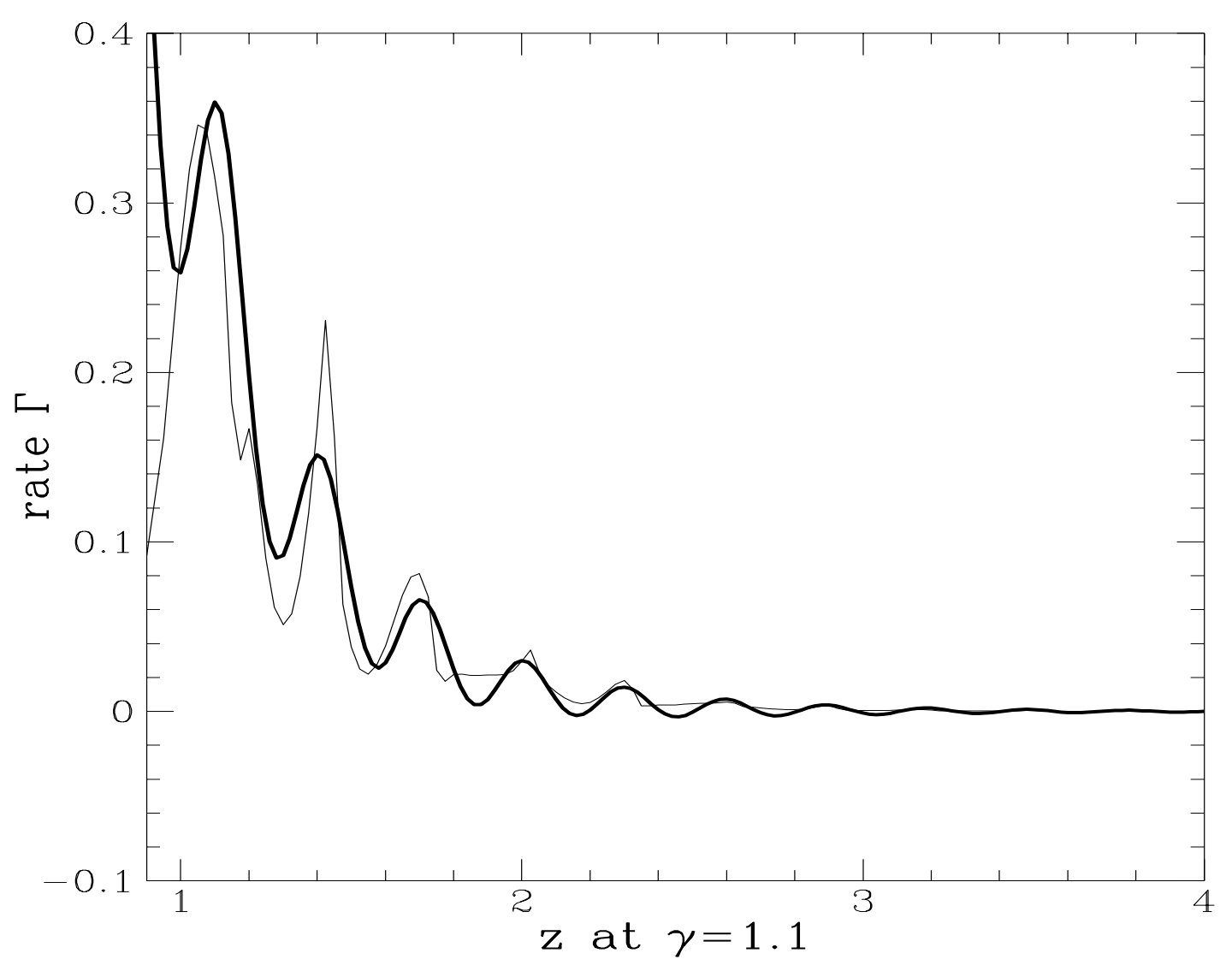

Figure 5: Numerical ionization rate (thin and jagged) and semiclassical approximation (thick and smooth) versus number of ponderomotive photons $z$ for $\gamma=1.1$

is fulfilled, the averaged WKB-value can be justified, and is in good agreement due to the very construction of our theory. For $\gamma \gg 1$, one should turn over to a pure multiphoton description [23, 24].

Figure 1 shows the numerical result (thin and jagged) for $\gamma=0.7$, together with the results of our theory. The background as well as the amplitude, phase, and periodicity of the modulation are very well comprised in the semiclassical theory for $z$ not too small.

The same is done in figure 5 for $\gamma=1.1$. Here again, one recognizes that the characteristic elements of the ionization curves are in good agreement. The same is valid of course for all other values $\gamma \approx 1$ and can be extended up to $\gamma \approx 2.5$, clearly beyond 


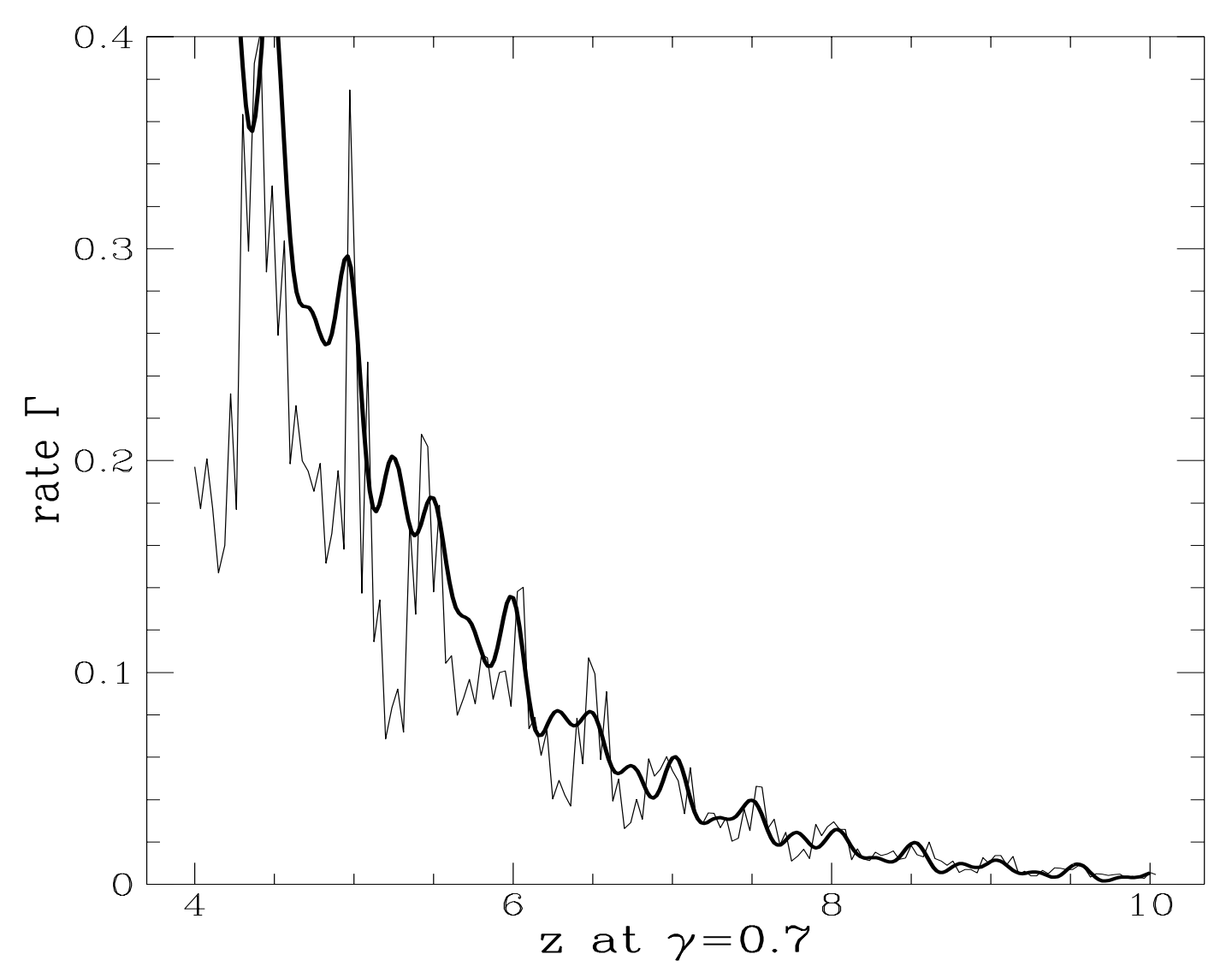

Figure 6: More fine-structure by considering longer periods than $2 \pi$, here two cycles $4 \pi$ considered

the adiabaticity regime.

If we want to incorporate more fine-structure superposed onto the modulation, we can consider longer periods than $2 \pi$. The result is a behaviour as in figure 6, which resembles closely the fine-structure, even if there can be no one-to-one correspondence between every small wiggle. Here the wave packets stemming from ionization bursts $\pi$ and $3 \pi$ were also taken into account, but they have very little influence on the result for $t_{f}=4 \pi$. The main contributions come of course from the wave packets stemming from $t=0$ and $t=2 \pi$. This can be seen as an example for the above qualititative statement about the relative importance of ionization at even or odd multiples of $\pi$.

The result (56) for fixed $\gamma$ and $z$ can easily be transcribed to other parameter combina- 


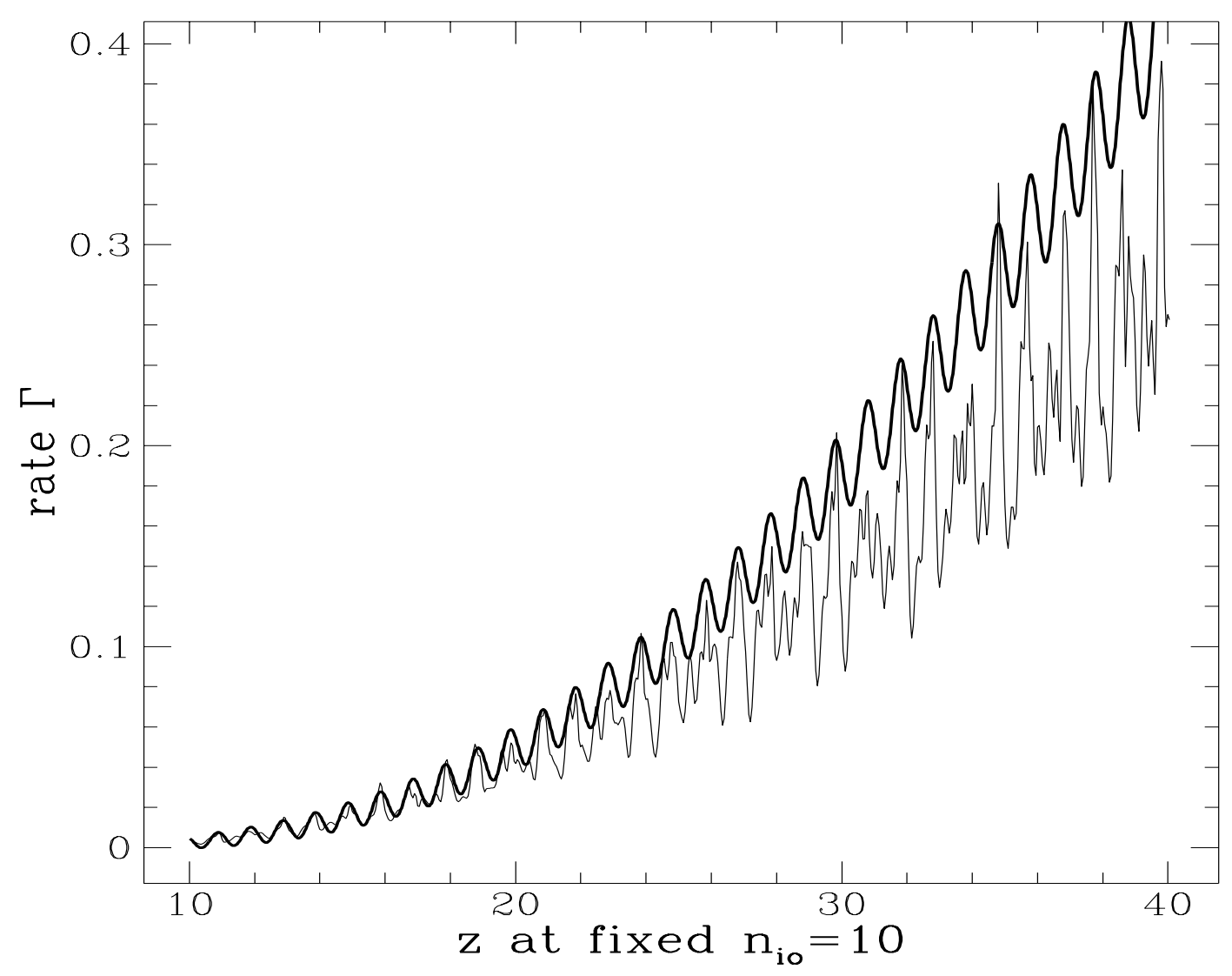

Figure 7: Now the depth of the binding potential is kept fixed, and $z$ is varied. The semiclassical theory (thick smooth curve) is compared to numerical results (thin and jagged).

tions. A common representation of ionization rates $\Gamma$ is to keep $n_{\text {io }}=2 \gamma^{2} z=\alpha^{2} /(2 \omega)$ fixed, i.e. the depth of the binding potential (last expression in atomic units as in section 2.1). One varies the intensity $\mu^{2}$ at fixed frequency $\omega$, which corresponds better to experimental situations. If one plots $\Gamma$ over the intensity or over $z=\mu^{2} /\left(4 \omega^{2}\right)$, one again obtains periodicity in the ponderomotive channel closing [26]. This is depicted in figure 7 in comparison with numerical data; note hereby that the semiclassical limit no more corresponds simply to $z \rightarrow \infty$, but is more involved and cannot be included simply into the representation. One again notices that the characteristic elements of ionization curves can be calculated in the semiclassical theory. 


\subsection{Comparison to other theories}

One major advantage and distinction of our theory from others (even from those claiming to be semi- or quasiclassic in some sense) is that we encounter no divergencies at channel closing thresholds, a result strongly supported by numerical evidence. Such divergencies typically occur in theories that (in our mind somehow arteficially) separate the ionization process into different channels, each one related to ionization by a distinct number of photons. Every time a channel closes, the corresponding ionization rate of the next higher channel (and so the overall rate) becomes infinite.

One earlier representative (eq.[31] by Perelomov, Popov, Terent'ev [13]; including quasiclassical features) and one more recent representative (eq.[44] by Susskind, Cowley, Valeo [14]; asymptotic in the number of photons for ionization) of this kind of theory is shown in figure 8 , in comparison with our result. The spikes in this figure are related to the closing of certain ionization channels with, say, $k$ photons at $z=z_{k}$ (eq. (10)), and they can be traced back to the divergence in the next higher channel with $k+1$ photons.

In between these thresholds, the background and the imposed modulation is approximately the same. But in contrast to these more implicit theories, where results are quite involved, our result (56,58) allows the direct, separate, and explicit evaluation of the background rate as well as of the amplitude and phase of the modulation. 


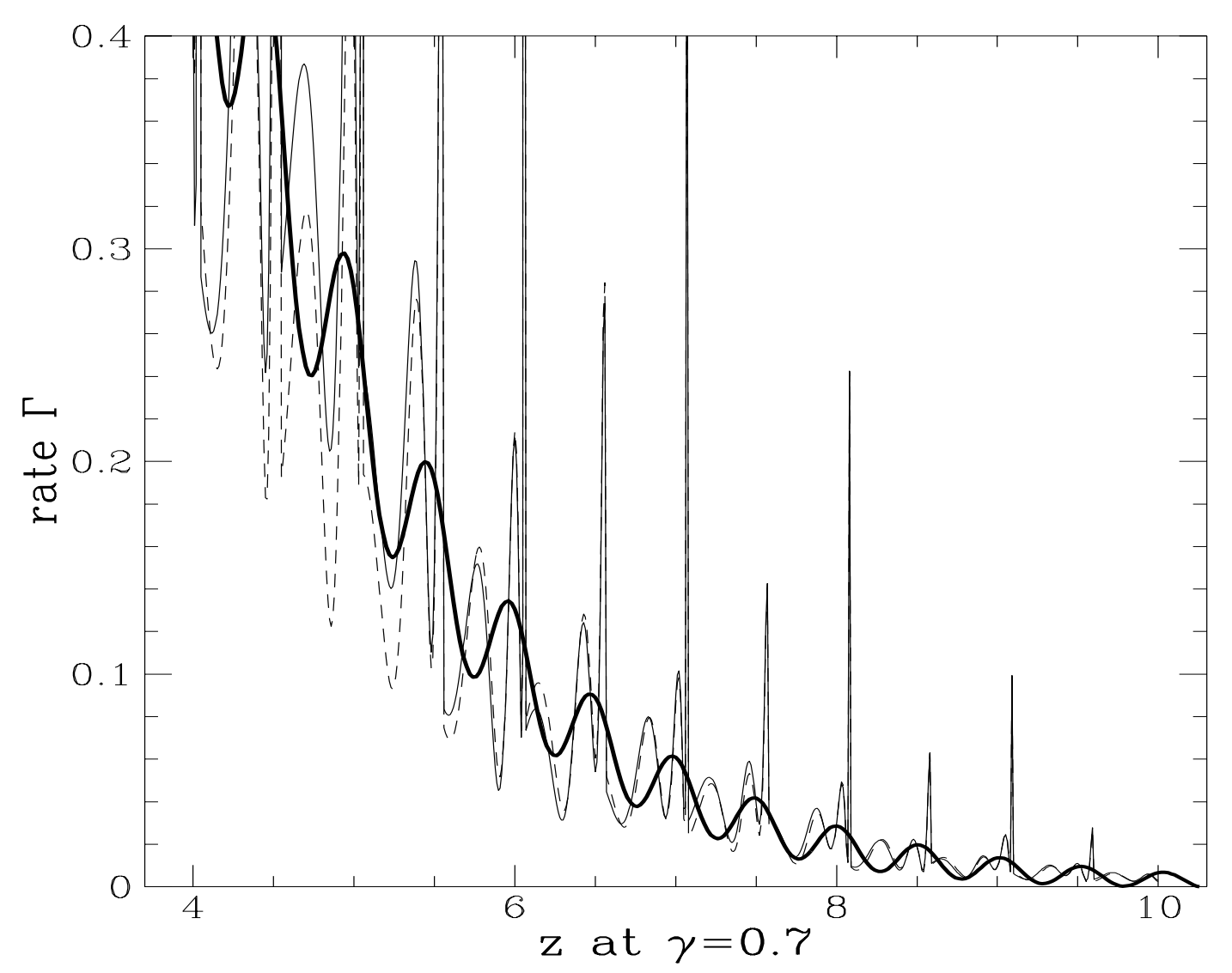

Figure 8: Comparison with other theories containing divergencies at channel closing thresholds: semiclassical theory (thick and smooth) versus Perelomov, Popov, Terent'ev [13] (thin line) and Susskind, Cowley, Valeo [14] (dashed), which nearly coincide in this region and especially in their behaviour at thresholds.

\section{Summary}

We succeeded in a semiclassical description of time-dependent tunneling and ionization in an oscillating field. The characteristic features of typical ionization curves can now be explained using a picture of tunneling, propagating and interfering wave packets (formula (56)). The main ingredients are first the separation of the ionization process into two disctinct steps, motivated by the asymptotic evaluation of instantaneous WKB-rates. And second the usage of complex time and analytical continuation of propagators, necessary to account for tunneling by a classical path description. The 
slow modulation with channel closing periodicity (an idea stemming from a multiphoton viewpoint) can be described correctly with respect to amplitude and to phase. Even the rich superposed fine-structure can be accounted for by considering a multitude of interfering wave packets.

\section{Acknowledgments}

The numerical calculations used for comparison were done with a program packet [1] kindly supplied by K. Sonnenmoser, whom is also thanked for two valuable discussions. G. Scharf is thanked for valuable discussions and suggestions at all stages of this work. This work was supported by Schweizerischer Nationalfonds. 


\section{A Saddle-Point Integration Techniques}

Under the notation of saddle-point integration, there exist two different versions in the literature (all considered in the limit $h \rightarrow 0$ ):

Method of steepest descent:

$$
\begin{array}{r}
\int g(t) \exp \left(-\frac{1}{h} f(t)\right) d t \approx \sqrt{\frac{2 \pi h}{\frac{\partial^{2}}{\partial^{2} t} f\left(t_{0}\right)}} g\left(t_{0}\right) \exp \left(-\frac{1}{h} f\left(t_{0}\right)\right) \\
\frac{\partial}{\partial t} f\left(t_{0}\right)=0, \frac{\partial^{2}}{\partial t^{2}} f\left(t_{0}\right)>0
\end{array}
$$

Here the argument in the exponent was expanded around the minimum $t_{0}$ of $f(t)$, up to second order, and the so created gaussian integral was evaluated exactly. If there exist several minima, one has to take the sum over them.

\section{Method of stationary phase:}

$$
\int g(t) \exp \left(\frac{i}{h} f(t)\right) d t \approx \sqrt{\frac{2 \pi i h}{\frac{\partial^{2}}{\partial t^{2}} f\left(t_{0}\right)}} g\left(t_{0}\right) \exp \left(\frac{i}{h} f\left(t_{0}\right)\right)
$$

This is in some respect the analytic continuation of (59); here every stationary point $t_{0}$ with $\partial_{t} f\left(t_{0}\right)=0$ is relevant, not only the minima.

\section{Analogy in functional integration:}

$$
\int_{t_{i}}^{t_{f}} \mathcal{D} x(t) g(x(t)) \exp \left(\frac{i}{h} S[x(t)]\right)
$$

In this path integral, one has to integrate over all possible paths with the boundary conditions

$$
x\left(t_{i}\right)=y, x\left(t_{f}\right)=x_{f}
$$

In the semiclassical approximation, one looks for the paths $x_{c l}$ which make the functional $S[x]$ stationary:

$$
\left.\delta S[x(t)]\right|_{x_{c l}}=0
$$

One again expands around these classical paths $x_{c l}$ (cf. e.g. [32]) and obtains the so-called Van-Vleck propagator [40], which in general must be corrected by so-called 
Maslov factors $\nu$ [33]. These take into account the crossing of caustics, i.e. divergencies of the mixed second derivative of $S^{c l}$.

$$
\begin{gathered}
\frac{1}{\sqrt{2 \pi i h}} \sqrt{-\frac{\partial^{2}}{\partial x_{f} \partial y} S^{c l}} \exp \left(\frac{i}{h} S^{c l}\right) \int_{t_{i}}^{t_{f}} g\left(x_{c l}(t)\right) d t \exp \left(-i \frac{\pi}{2} \nu\right) \\
x_{c l}\left(t_{i}\right)=y, x_{c l}\left(t_{f}\right)=x_{f},\left.\delta S[x(t)]\right|_{x_{c l}}=0, S^{c l}=S\left[x^{c l}\right]
\end{gathered}
$$

\section{B Semiclassical Propagator using the WKB-Ansatz}

This is an alternative to the construction of the semiclassical propagator $U^{s c}\left(x, t ; y, t_{i}\right)$ by the path intgral approach (cf. section 5.1 and appendix А). We construct $U^{s c}\left(x, t ; y, t_{i}\right)$ in such a way that it fulfills the time-dependent Schrödinger equation (4) with respect to $t$ and $x$ up to $O\left(h^{2}\right)$.

$$
\begin{aligned}
i h \frac{\partial}{\partial t} U^{s c}=\hat{H} U^{s c} & =\left(-\frac{1}{2} h^{2} \frac{\partial^{2}}{\partial x^{2}}-h \gamma \delta(x)-x \cos (t)\right) U^{s c} \\
\lim _{t \rightarrow t_{i}} U^{s c}\left(x, t ; y, t_{i}\right) & =\delta(x-y)
\end{aligned}
$$

We make the ansatz

$$
U=\varphi\left(x, t ; y, t_{i}\right) \exp \left(\frac{i}{h} S\left(x, t ; y, t_{i}\right)\right)
$$

and yield

$$
i h \partial_{t} \varphi-\varphi \partial_{t} S=\frac{1}{2}\left(\partial_{x} S\right)^{2}-i h \partial_{x} \varphi \partial_{x} S-\frac{i h}{2} \varphi \partial_{x}^{2} S-\frac{h^{2}}{2} \partial_{x}^{2} \varphi-h \gamma \delta(x) \varphi-x \cos (t) \varphi
$$

Comparing the distinct orders of $h$ :

$$
\begin{aligned}
h^{0} \quad: \quad 0=\partial_{t} S+\frac{1}{2}\left(\partial_{x} S\right)^{2}-x \cos (t) \\
h^{1}: \quad i \gamma \delta(x) \varphi=\partial_{t} \varphi+\partial_{x} \varphi \partial_{x} S+\frac{1}{2} \varphi \partial_{x}^{2} S \\
2 i \gamma \delta(x) \varphi^{2}=\frac{\partial}{\partial t} \varphi^{2}+\frac{\partial}{\partial x}\left(\varphi^{2} \partial_{x} S\right)
\end{aligned}
$$

The right-hand side of equation (73) is just a conservation equation with density $\varphi^{2}$ and flow $\varphi^{2} \partial_{x} S$, which is fulfilled everywhere except the origin. The equation (71) for $h^{0}$ is easily solvable, it is just the Hamilton-Jacobi equation $-\partial_{t} S=H\left(x, \partial_{x} S\right)$ for the 
motion of an electron in an oscillating electric field (with Lagrangian $L_{0}$ ). One can easily solve the appropriate equation of motion and yields the classical path $x_{c l}(\tau)$ with boundary conditions $x_{c l}(t)=x$ and $x_{c l}\left(t_{i}\right)=y$.

$$
x_{c l}(\tau)=-\cos (\tau)+\cos \left(t_{i}\right)+y+\frac{x-y+\cos (t)-\cos \left(t_{i}\right)}{t-t_{i}}\left(\tau-t_{i}\right)
$$

The action $S$ is then the time integral over the classical Lagrangian $L_{0}$ :

$$
S=\int_{t_{i}}^{t} L_{0}\left(x_{c l}\left(t^{\prime}\right), \dot{x}_{c l}\left(t^{\prime}\right)\right) d t^{\prime}
$$

Then, as usual, the expression $\varphi_{0}=\sqrt{-\partial_{x} \partial_{y} S}=1 / \sqrt{t-t_{i}}$ solves the equation (72) for $h^{1}$ outside the origin. Now we make the ansatz

$$
\varphi=\beta\left(x, t ; y, t_{i}\right) \varphi_{0}=\frac{\beta}{\sqrt{t-t_{i}}}
$$

Inserting this in (72), we yield

$$
i \gamma \delta(x) \beta=\partial_{t} \beta+\partial_{x} \beta \partial_{x} S
$$

This linear partial differential equation of first order can be solved using the method of characteristics. The (ordinary) differential equation for the characteristic $x_{T}$ is

$$
\frac{d}{d t} x_{T}=\partial_{x} S\left(x, t ; y, t_{i}\right)
$$

But $S$ is, as we know, the action for the classical path and therefore $\partial_{x} S$ is just the momentum of the classical path $\partial_{x} S=\dot{x}_{c l}(t)$ (e.g. [41). So we conclude that the characteristic is just the classical path $x_{T}=x_{c l}$. We obtain the following (ordinary) differential equation for $\beta$ :

$$
\left.\frac{d}{d t} \beta\right|_{x_{c l}}=\partial_{t} \beta+\partial_{x} \beta \frac{d}{d t} x_{c l}=\left.i \gamma \delta(x) \beta\right|_{x_{c l}}
$$

This can be integrated straight forward and the result is

$$
\left.\beta(t)\right|_{x_{c l}}=\exp \left(i \gamma \int_{t_{i}}^{t} \delta\left(x_{c l}\left(t^{\prime}\right)\right) d t^{\prime}\right) \beta_{0}
$$


This again can be understood as a phase jump for every crossing of the $\delta$-potential at the origin; the constant $\beta_{0}$ is fixed to $\beta_{0}=1 / \sqrt{2 \pi i h}$ by the required normalization (68) of $U^{s c}$.

To summarize, we end up with the following result, identical to eq. (35)

$$
U^{s c}\left(x, t ; y, t_{i}\right)=\frac{1}{\sqrt{2 \pi i h\left(t-t_{i}\right)}} \exp \left(\frac{i}{h} S\left[x_{c l}\right]\left(x, t ; y, t_{i}\right)\right) \exp \left(i \gamma \int_{t_{i}}^{t} \delta\left(x_{c l}\left(t^{\prime}\right)\right) d t^{\prime}\right)
$$

\section{Complex Time Description of Tunneling Processes}

The usefulness of a complex time coordinate for describing tunneling processes is best demonstrated by a simple example. Consider an inverse harmonic potential barrier $V(x)=-x^{2} / 2$ and a particle coming in from $-\infty$ with energy $E=-1 / 2$. The classical equation of motion $\ddot{x}_{c l}=x_{c l}$ is fulfilled by the classical trajectory $x_{c l}(t)=-\cosh (t)$, restricting the particle to $x_{c l} \leq-1$.

In this time-independent problem, the total energy $E$ is a constant of motion:

$$
E=\frac{\dot{x}^{2}}{2}-\frac{1}{2} x^{2}=-\frac{1}{2}
$$

This differential equation can be solved for $t(x)$ :

$$
t(x)= \pm \int_{-1}^{x} \frac{d x^{\prime}}{\sqrt{2 E+x^{\prime 2}}}
$$

The range of $x$ can now be extended formally to $x>-1$, then $t$ acquires an imaginary part for $x^{\prime} \in[-1,1]$

$$
\int_{-1}^{1} \frac{d x^{\prime}}{\sqrt{2 E+x^{\prime 2}}}=i \pi
$$

The tunneling trajectory through the barrier can be described in the complex $t$-plane by the path $[-\infty, 0],[0, i \pi],[i \pi,+\infty+i \pi]$. The second part is the tunneling process through the barrier, noting that

$$
x_{c l}(i \tau)=-\cosh (i \tau)=-\cos (\tau)
$$

The third part describes the propagation on the other side of the barrier, noting that

$$
x_{c l}(t+i \pi)=-\cosh (t+i \pi)=+\cosh (t)
$$




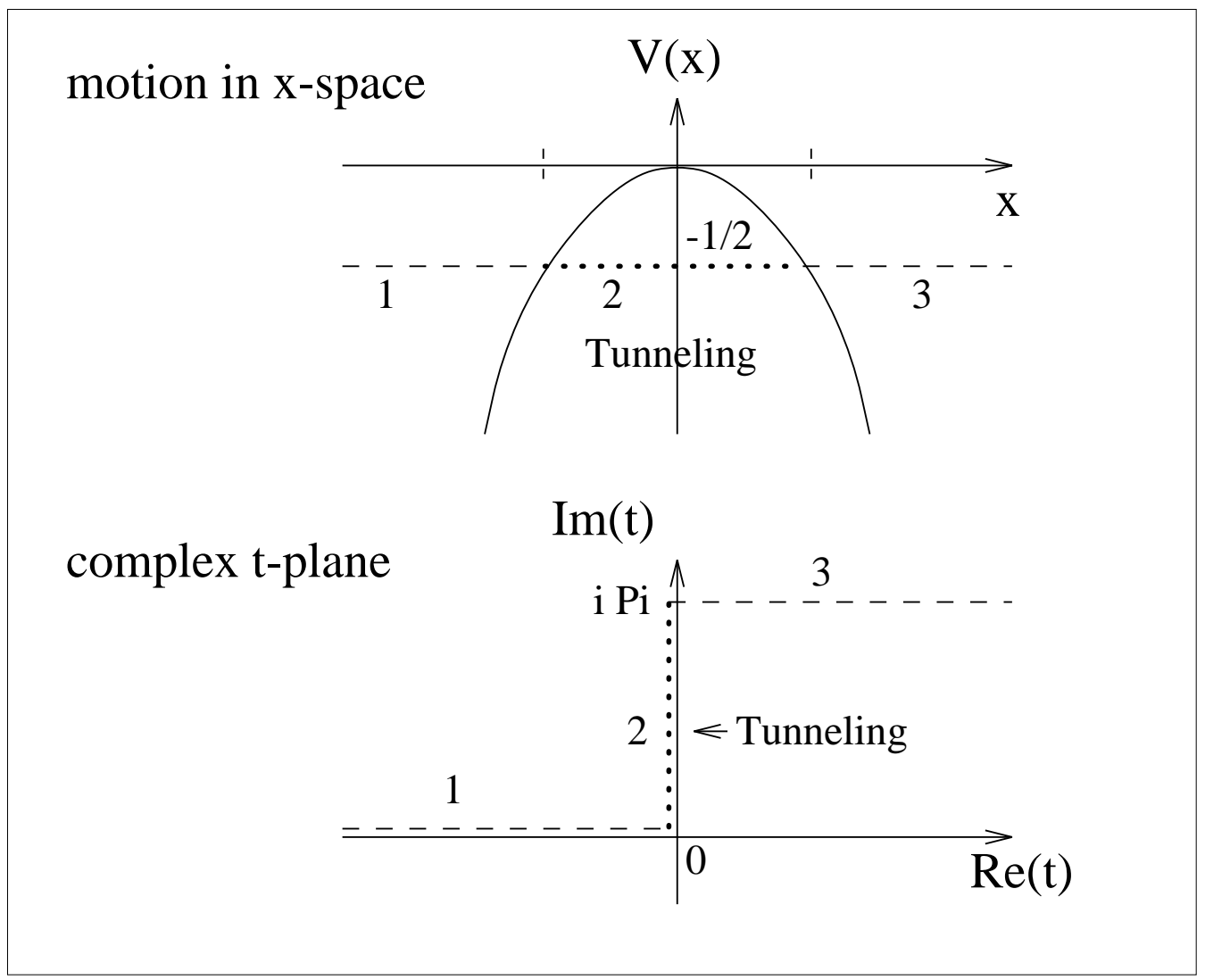

Figure 9: Tunneling in $x$-space and the appropriate complex time path for an inverse harmonic potential

Figure 9 illustrates this by contrasting the path in $x$-space to the path in the complex $t$-plane. For mathematical foundations of the analytic continuation applied above see [35], for recent applications of this method compare e.g. [36, 42]. 


\section{Extensibility and Comparison to other Models}

In section 2.2, there was explicitly made use of the scaling property $\delta(\beta x)=\delta(x) / \beta$ (for $\beta>0$ ) of the one-dimensional $\delta$-function. For the Coulomb-potential $V_{C}$, the scaling behaviour is identical, so that the whole calculation can be repeated. The main difference consists in a different phase factor $\phi_{C}$ (cf. (32)), which is now the line integral over the Coulomb-potential:

$$
\phi_{C}=\int_{t_{i}}^{t_{f}} V_{C}\left(x_{c l}(t)\right) d t
$$

This is due to the fact that the binding potential is suppressed by a factor $h$ in the transformed Schrödinger equation (4). As derived in sections 5.1 and appendix B, such a suitably suppressed binding potential does not influence the classical trajectories, but only changes the phase transported along these trajectories by an additional phase factor $\phi_{C}$ (given above).

This separation is no longer valid for other types of potentials with different (or without) scaling properties. Here the classical equations of motion must be solved fully for the binding potential plus electromagnetic field. In the time-dependent case, there generally does not exist a first integral of motion (like the energy in the static case), and so the classical system is not integrable in closed form.

The physics of the ionization process should remain the same: complex time-dependent tunneling followed by free propagation (two-step models). This is reflected by the fact that ionization curves for other model potentials show qualitatively the same characteristics. The semiclassical "sum over classical paths"-method claims to work in this case anyway, but unfortunately this cannot be done in analytical form because of the nonintegrability already on the classical level. 


\section{References}

[1] G. Scharf, K. Sonnenmoser, W.F. Wreszinski, Phys. Rev. A 44, 3250 (1991).

[2] G. Mainfray, C. Manus, Rep. Prog. Phys. 54, 1333 (1991).

[3] K. Burnett, V.C. Reed, P.L. Knight, J. Phys. B: At. Mol. Opt. Phys. 26, 561 (1993).

[4] J.H. Eberly, J. Javanainen, K. Rzazewski, Phys. Rep. 204, 331 (1991).

[5] Atoms in Intense Laser Fields, edited by M. Gavrila (Academic, Orlando, 1992).

[6] special issue of J. Opt. Soc. Am. B 7, (4, 1990).

[7] K. Sonnenmoser, J. Phys. B: At. Mol. Opt. Phys. 26, 457 (1993).

[8] H. Massey, Negative Ions (Cambridge University Press, Cambridge, 1976).

[9] L. Keldysh, Sov. Phys. JETP 20, 1307 (1965).

[10] F. Faisal, J. Phys. B 6, L89 (1973).

[11] H. Reiss, Phys. Rev. A 22, 1786 (1980).

[12] D. Volkov, Z. Phys. 94, 250 (1935).

[13] A.M. Perelomov, V.S. Popov, M.V. Terent'ev, Sov. Phys. JETP 23, 924 (1966).

[14] S.M. Susskind, S.C. Cowley, E.J. Valeo, Phys. Rev. A 42, 3090 (1990).

[15] W. Becker, R.R. Schlicher, M.O. Scully, J. Phys. B 19, L785 (1986).

[16] K.C. Kulander, K.J. Schafer, J.L. Krause, in Proceedings of the Workshop Super Intense Laser Atom Physics SILAP III, NATO Advanced Study Institute Series $B$, edited by B. Piraux et al (Plenum Press, New York, 1993).

[17] P. Corkum, Phys. Rev. Lett. 71, 1994 (1993). 
[18] M. Lewenstein, Ph. Balcou, M.Y. Ivanov, A. L'Huillier, P.B. Corkum, Phys. Rev. A 49, 2117 (1994).

[19] J. Shirley, Phys. Rev. B 138, 979 (1965).

[20] S. Chu, Adv. At. Mol. Phys. 21, 197 (1985).

[21] R.M. Potvliege, R. Shakeshaft, Phys. Rev. A 38, 4597 (1988).

[22] W. Becker, J.K. McIver, M. Confer, Phys. Rev. A 40, 6904 (1989).

[23] N.B. Delone, V.P. Krainov, Multiphoton processes in atoms (Springer, New York, 1994).

[24] F. Faisal, Theory of Multiphoton Processes (Plenum Press, New York, 1987).

[25] W.H. Press, S.A. Teukolsky, W.T. Vetterling, B.P. Flannery, Numerical Recipes, second edition (Cambridge University Press, Cambridge, 1992).

[26] J. Eberly, J. Phys. B: At. Mol. Opt. Phys. 23, L619 (1990).

[27] W.G. Greenwood, J.H. Eberly, Phys. Rev. A 43, 525 (1991).

[28] M. Gutzwiller, Chaos in Classical and Quantum Mechanics (Springer, New York, 1990).

[29] W. Elberfeld, M. Kleber, Z. Phys. B 73, 23 (1988).

[30] M. Pont, R. Shakeshaft, R.M. Potvliege, Phys. Rev. A 42, 6969 (1990).

[31] S. Geltman, J. Phys. B. 11, 3323 (1978).

[32] L. Schulman, Techniques and Applications of Path Integration (John Wiley \& Sons, USA, 1981).

[33] V.P. Maslov, M.V. Fedoriuk, Semi-classical Approximation in Quantum Mechanics ( D. Reidel Publishing Company, Dordrecht, 1981). 
[34] R.P. Feynman, A.R. Hibbs, Quantum mechanics and Path Integrals (McGrawHill, USA, 1965).

[35] D. McLaughlin, J. Math. Phys. 13, 1099 (1972).

[36] M. Child, Semiclassical Mechanics with Molecular Approximations (Clarendon Press, Oxford, 1991).

[37] V.S. Popov, V.P. Kuznetov, A.M. Perelomov, Sov. Phys. JETP 26, 222 (1968).

[38] M.V. Berry, K.E. Mount, Rep. Prog. Phys. 35, 315 (1972).

[39] A. Voros, Ann. Inst. Henri Poincaré XXIV, 31 (1976).

[40] J. van Vleck, Proc. Natl. Acad. Sci. USA 14, 178 (1928).

[41] H. Goldstein, Classical Mechanics (Addison-Wesley, Cambridge, Mass., 1950).

[42] M. Kira, I. Tittonen, W.K. Laii, S. Stenholm, Phys. Rev. A 51, 2826 (1995). 